
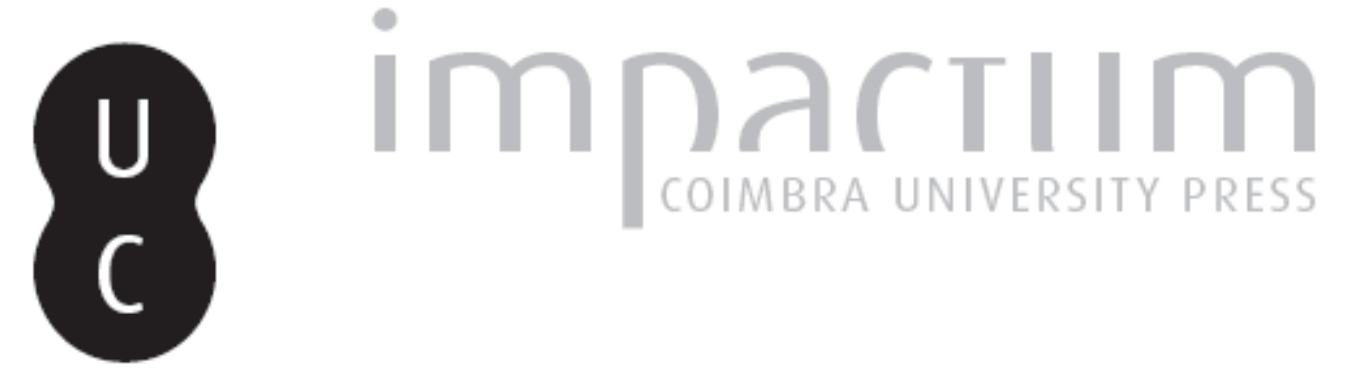

\title{
Sul conceito teologico di tempo nella dimensione di mondo
}

\section{Autor(es): $\quad$ Longo, Gianfranco}
Publicado por: Universidade Católica de Petrópolis; Instituto Brasileiro de Informação em Ciência e Tecnologia

URL persistente:

URI:http://hdl.handle.net/10316.2/33000

DOI:

DOI:http://dx.doi.org/10.14195/1984-6754_4-1_2

Accessed : $\quad$ 26-Apr-2023 04:51:50

A navegação consulta e descarregamento dos títulos inseridos nas Bibliotecas Digitais UC Digitalis, UC Pombalina e UC Impactum, pressupõem a aceitação plena e sem reservas dos Termos e Condições de Uso destas Bibliotecas Digitais, disponíveis em https://digitalis.uc.pt/pt-pt/termos.

Conforme exposto nos referidos Termos e Condições de Uso, o descarregamento de títulos de acesso restrito requer uma licença válida de autorização devendo o utilizador aceder ao(s) documento(s) a partir de um endereço de IP da instituição detentora da supramencionada licença.

Ao utilizador é apenas permitido o descarregamento para uso pessoal, pelo que o emprego do(s) título(s) descarregado(s) para outro fim, designadamente comercial, carece de autorização do respetivo autor ou editor da obra.

Na medida em que todas as obras da UC Digitalis se encontram protegidas pelo Código do Direito de Autor e Direitos Conexos e demais legislação aplicável, toda a cópia, parcial ou total, deste documento, nos casos em que é legalmente admitida, deverá conter ou fazer-se acompanhar por este aviso.

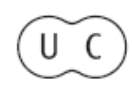



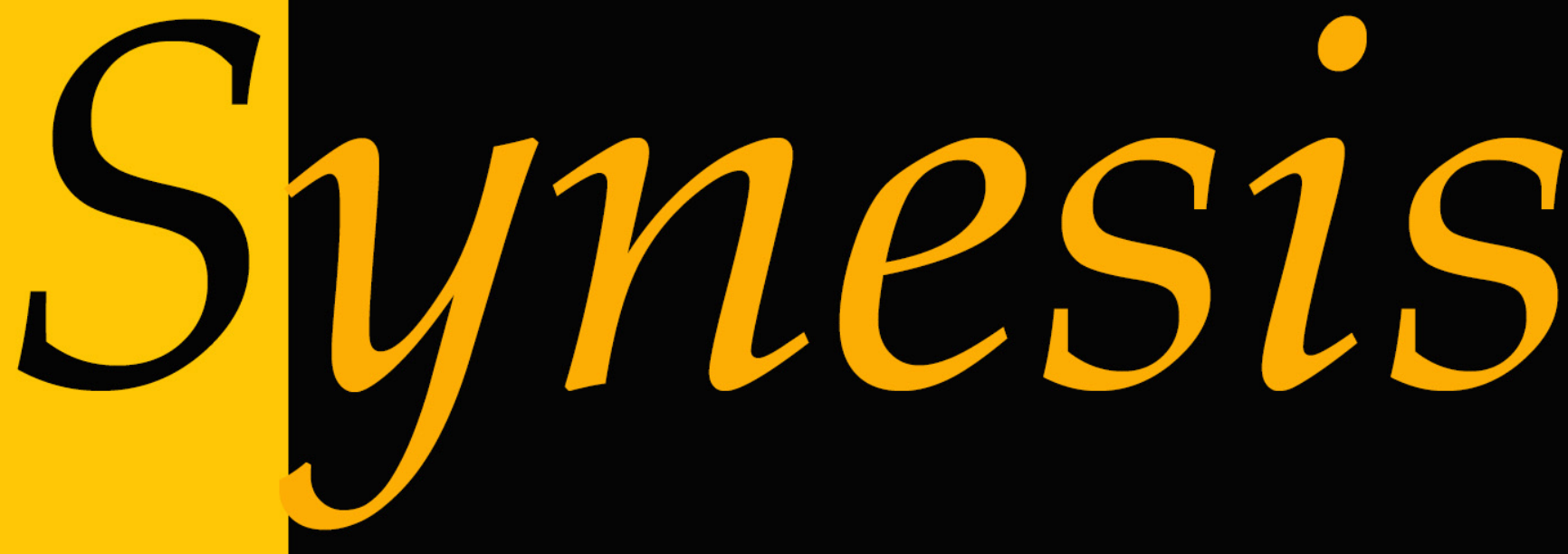

Revista do Centro de Teologia e Humanidades ISSN 1984-6754

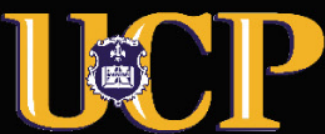




\title{
SUl CONCETTO TEOLOGICO DI TEMPO NELLA
}

\section{DIMENSIONE DI MONDO'}

Gianfranco Longo $^{2}$

\begin{abstract}
La domanda circa il tempo vuole definire la differenza tra un tempo teologico ed uno del mondo. Esattamente nel tempo come circostanza (каıрós) emerge la condizione del mondo nel tempo; nella dimensione del tempo come epoca e storia ( $\chi \rho o ́ v o \varsigma$ ) si coglie il significato del tempo-mondo; mentre nell'aíóv si determina la pluralità del tempo nel mondo, cioè l'eternità di Dio. Infatti solo Dio unitariamente è, perché unicamente accaduto e umanamente rivelatosi in Cristo; e l'uomo a sua volta diviene realizzandosi nell'esistere di Cristo, perché uomo che rivela Dio, accadendo Gesù in Dio e vivendo, Lui Cristo uomo, una perfetta natura divina creatrice.
\end{abstract}

Parole Chiave: Tempo teologico; Mondo; Eternità di Dio.

Abstract: The question about the time tries to seattle the difference between a theological time and a world time. Exactly in the time as circumstance (каı)́s) stands out the world's

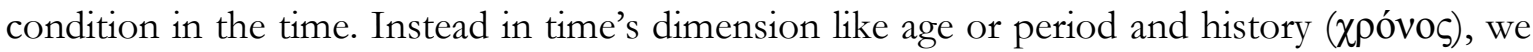
can seaze the time-world's meaning; while in the aíw the time's plurality in the world determines itself: i.e. we have the Eternity of God. In fact only God jointly is, because once happened and in Christ himself once rivelated too. The man every time becomes when he realizes himself in the Christ's life, because Christ is the true Man who bares God, becoming Jesus in God and living as Man a perfect nature which is divine and creative too.

Keywords: Theological time; World; Eternity of God.

\footnotetext{
${ }^{1}$ Ricevuto articolo 18/06/2012 ed accettato per pubblicazione da parte del Comitato Editoriale di 05/07/2012.

2 Gianfranco Longo (Bari, 1965), ha studiato alcuni anni nella Westfählische-Wilhelms Universität di Münster, conseguendo un dottorato in Sociologia del diritto (1994) e successivamente un dottorato in Diritto costituzionale (2006). Dopo aver insegnato sia Diritto costituzionale che Diritto costituzionale comparato nella Facoltà di Scienze Politiche dell'Università di Bari, è attualmente docente, nella stessa Università di Bari, di Teoria generale del diritto e di Filosofie comparate.

Email: gianfranco.longo@,uniba.it.
} 


\section{Sul concetto di tempo.}

La riflessione su un concetto comporta, parallelamente, una definizione non sempre univoca, piuttosto plurale in prospettiva semantica, giacché il concetto, quale che esso sia, è presente nella storia trasformandosi tuttavia nella sua stessa storia, assumendo una molteplicità di semantiche adeguate a quelle determinate epoche in cui quello specifico concetto possa esser stato particolarmente significativo.

Il concetto pertanto si semanticizza nel corso del "tempo", profilando una sua propria storia.

Quando poi si vuol definire o comunque riflettere proprio sul concetto di tempo, che a sua volta caratterizza la stessa semantica storica perché è nel tempo che si prospettano evoluzioni e variabilità delle interpretazioni di un determinato concetto, diviene impossibile delineare una categoria semantica univoca e invariabile: il tempo caratterizza il mondo come

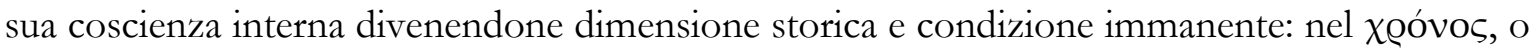

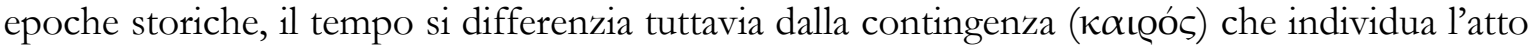

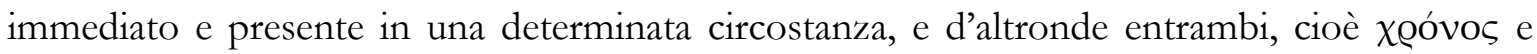

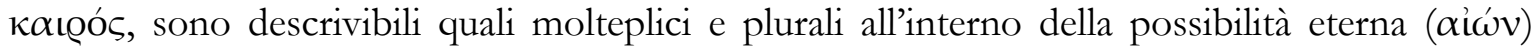
della tradizione e della trasformazione, nonché rinascita, di un concetto in semantiche sempre differenziabili.

Peraltro a seconda delle diverse epoche in cui si compara e si analizza uno specifico concetto andandolo quindi effettivamente a storicizzare, il tempo esprime un richiamo all'indecidibilità gödeliana: posso certamente percepire la mia origine, esserne convinto e sicuro, posso anche stabilire il punto d'inizio del mio tempo, per cui esisto; ciononostante essere costretti a non poter definire il luogo di arrivo (la mia morte) e il suo proprio spazio e "tempo" (in tal caso kaı@ós), mi riporta indietro, obbligandomi ad accettare sia la questione cosmologica, come anche quella ontologica, plausibili nella generazione e nella corruzione che unicamente il Tempo indica quale movimento caratterizzante dell'universo e dell'uomo: esisto ma non sono, perché sarei se, e solo se, fossi a conoscenza del mio stesso intero percorso esistenziale,

\footnotetext{
${ }^{3}$ Su questi e altri profili critici si permetta di rinviare a G. LONGO, Trattato di semantica storica e di logica giuridica costituqionale, Aracne, Roma 2009, soprattutto a quelle parti concernenti l'analisi della semantica storica quale programma di critica della conoscenza, e a quelle teoretiche di definizione del concetto tout court. Si veda ibid. pp. 35 e ss.
} 
percorso che diviene possibile e completamente raggiungibile nell'esser compreso al momento paradossale della fine di questo cammino: cioè la propria morte.

Ragion per cui solo Dio unitariamente è, perché unicamente accaduto e umanamente rivelatosi in Cristo; e l'uomo a sua volta diviene realizzandosi nell'esistere di Cristo, perché uomo che rivela Dio, accadendo Gesù in Dio e vivendo, Lui Cristo uomo, una perfetta natura divina creatrice.

\section{La misura del prima e del dopo: il movimento.}

La "supremazia" del Tempo, allora, quale indecidibile parte del movimento e dell'origine dell'universo, parve essere fondata già ad Aristotele che così sosteneva: "Inoltre, com'è possibile che vi siano 'prima' e 'dopo', senza che esista tempo? O il tempo, senza che vi sia movimento? Se dunque il tempo è numero del movimento o un certo movimento, e se il tempo è eterno, necessariamente anche il movimento sarà eterno" ${ }^{4}$.

Quindi proprio nell'istante, prosegue Aristotele, si rivela l'esserci del Tempo parte del mio stesso esistere. Questo mio esistere perciò può sì evidenziarsi ed emergere dalla misura del Tempo, ugualmente però tale misura, quella cioè della mia vita, rimane ignota al mio essere: poiché soltanto nel mondo il mio esistere si commisura al mio tempo, parte di quello del mondo, cioè temporalită 5 .

In questa temporalità, nella parte del Tempo che caratterizza quella parte del mondo che io occupo, "vivono" a loro volta le temporalizzazioni del mio esistere, cioè le pluralità e le variabilità delle forme della mia vita all'interno del mondo, però come elementi discreti di questo mondo in grado di specificare singoli passaggi esistenziali, particolari istanti nell'ambito di un determinato periodo.

Ecco perché il movimento parve ad Aristotele comprovare l'eternità del Tempo in relazione all'universo: l'osservazione del mondo secondo quanto il Tempo determina la dimensione del mondo, non sarebbe altro che misura dello Spazio in cui questo movimento accade, divenendo tale osservazione a-posteriori una descrizione dell'ordine in cui tutte quante le

\footnotetext{
${ }^{4}$ Cfr. ARISTOTELE, Fisica, a cura di Luigi Ruggiu, Mimesis Edizioni, Milano 2007, p. 321.

5 In Heidegger il rilievo dato al concetto di temporalità assume una schematizzazione che trascende il mondo; in ciò la temporalità mondanizza il mondo, rendendo il Tempo essere-nel-mondo. Cfr. M. HEIDEGGER, Essere e tempo, a cura di Alfredo Marini, Mondadori, Milano 2006, pp. 987 e ss.
} 
cose avvengono nel mondo, e nel suo spazio, in base al Tempo del mondo: кóouos infatti in greco ha come suo primo significato quello di "ordine".

Tuttavia all'interno del mondo si enuclea un'eidetica Tempo: la forma enuncia solo l'apparenza del fenomeno. Si avrà così una peculiare forma del Tempo che successivamente darà luogo al fenomeno del mondo, perché ontico nel Tempo che caratterizza e che lo caratterizza: Heidegger nella relazione Tempo-Mondo rilevava piuttosto l'esserci (Dasein) che avrebbe permesso la consistenza ontologica del mondo.

Si vuole d'altro canto rimarcare come, senza mondo, il Tempo non avrebbe ragion d'essere, sarebbe mera infinità, indeterminabile paradossalmente anche nel finito. Solo nel mondo però il Tempo acquisisce una singolare dimensione di misura, proprio perché il mondo è mosso dal Tempo, eppure sospinto da ciò che non ha avuto inizio perché non potrebbe aver fine.

\section{L'onticità del tempo: Cristo nel mondo.}

In tale prospettiva il mondo si rivela anche onticità all'interno del Tempo che consta tuttavia di elementi discreti (intesi come interruzioni e cesure riconducibili alle temporalità e alle temporalizzazioni), i quali per il loro stesso interrompere la durata, che non avrebbe misura dell'eternità, trascendono la connessione Tempo-Mondo consentendo il formarsi dell'entemondo distaccato dall'ente-tempo.

Ciò consente di oltrepassare quanto, proprio Heidegger, profilava nel concetto di ente intramondano (das innerweltliche Seiende).

Pertanto l'indipendenza dell'ente-mondo dall'ente-tempo valorizzerebbe l'elemento discreto nell'essere del tempo e nell'essere del mondo, coniugando però onticamente il Tempo del mondo al mondo vissuto, che possiede una sua forma peculiare nella storia del Tempo stesso.

In ciò ci aiuta Heidegger quando nota che la temporalità (die Zeitlichkeit) esistenzialmente si "disvela" nella temporalizzazione (die Zeitigung o anche die Verzeeitlichung), definendo infine una connessione Tempo-Mondo evidente da un punto di vista esistenziale, però onticamente possibile solo in una frattura della forma continua da parte dell'elemento discreto caratterizzante il divenire del Tempo nel mondo e di quest'ultimo all'interno della sua stessa mondanità (die Weltlichkeit). 
In buona sostanza ci si vuol riferire all'esperienza del mondo all'interno del suo stesso Tempo, poiché formazione di temporalità e di temporalizzazione ${ }^{6}$.

In tale senso Dio imprime movimento al mondo e rivela il mondo come specificato dal Tempo: il Tempo meccanicizza razionalmente il divenire del mondo e Dio esiste solo in Cristo, rivelandosi ed essendosi rivelato nel Tempo del mondo: Cristo è eterno perché Dio per il Tempo e per il mondo, che avviene nel mondo, avvenimento suo proprio di Cristo, quale imprescindibile suo dover essere eterno ${ }^{7}$.

Si profila completa in tal modo l'onticità stessa della tradizione del mondo, in quanto la trasformazione si rivela all'interno di un'unità temporale che fa della tradizione il suo fondamento. Il mondo nel suo sviluppo ontico (l'onticizzazione) raffigura suoi propri mutamenti attraverso le fasi di temporalizzazione del Tempo e nella sua onticità, cioè nelle temporalità che delineano il mondo quale movimento nel Tempo e che storicizzano il mondo quale divenire del suo peculiare dover-essere-mondo. Così la temporalità presente pone la memoria del passato come variabile probabilità dell'accadere futuro e trasformazione proprio dell'essere-presente quale sviluppo nel dopo; un dopo che naturalmente non potrebbe avere un prima, non potendo ancor essere consci e certi di quanto accadrà in quel determinato dopo.

Infatti solo ciò-che-è-avvenuto delimita la determinatezza del dopo: è come se fossimo disposti a ricostruire l'intero assetto del Tempo unicamente nel suo specifico passato, osservandone gli istanti peculiari di evoluzione del passato nel passato, poi nel presente e infine nel futuro rappresentato da quel mondo passato e memorizzato come storico, cioè ormai storicizzato: ragion per cui si peculiarizza la storia del Tempo nel mondo come misura dell'avvenimento del mondo nel rapporto spazio-tempo ${ }^{8}$.

\footnotetext{
${ }^{6}$ Si desidera ancora rinviare a M. HEIDEGGER, cit., pp. 199 e ss. e 993 e ss.

${ }^{7}$ Dice Blumenberg: "Solo, muoversi non è necessario a un Dio. Egli desume la sua opera dal modello che ruota, appunto non 'leggendo'. Aspetto per aspetto, egli trae dal prototipo uno stato dell'universo dopo l'altro, ognuno dei quali sta per una sostanza rappresentante e la parte che le spetta del tutto, la cui dimensione temporale costituisce appunto la storia”, così H. BLUMENBERG, La leggibilità del mondo, Il Mulino, Bologna 1984, p.123. 8 Jean Paul Sartre, ne L'essere e il nulla, individua il nocciolo di questa problematica quando dice: "Noi conserviamo sempre la possibilità di cambiare il significato del passato, in quanto esso è un ex-presente, che ha avuto un avvenire", così J.- P. SARTRE, L'essere e il nulla. Saggio di ontologia fenomenologica, Il Saggiatore, Milano 1984, p. 164. Sartre però non indugia nelle pagine che seguono sulla parte relativa alla Temporalità, del suo famoso saggio, a rilevare come in fondo non si possa più essere il proprio passato, proprio perché lo si era consegnando dunque alla dimensione presente la stima del precedente, senza che questa assuma però il carattere di una condizione permanente nel futuro.
} 
Da ciò si chiarisce il dilemma umano tra vita e morte, ma soprattutto si definisce l'aporia della vita che ha come meta il suo contrario: André Malraux sostenne acutamente: "Ciò che vi è di terribile nella morte, è che trasforma la vita in destino"".

La morte fa sì che la vita raggiunga il suo traguardo, cioè raggiunga in-sé ciò che non sarebbe-per-sé, ciononstante ineluttabilmente presente della vita stessa, minaccia costante che constata vitale il presente eppure irraggiungibile il passato, aporia che rappresenta, altrettanto efficacemente, quanto il futuro non abbia modo di possibilizzarsi nel mio presente come aspettativa di quel presente che trascina, in realtà, un passato ricco di aspettative che erano ma che non possono più essere, rinvio a un futuro che è già un deludente presente. Tutte queste interruzioni del tempo, i passaggi dal passato al presente al futuro, permettono tuttavia l'affidabile costanza del mondo.

Pertanto sebbene l'unità del movimento del Tempo non presuma una sua specifica continuità, mostrando piuttosto l'oggettività storica di quanto interrotto, il mondo si ridetermina nellinterruzione come effetto di trasformazione, per essere un susseguirsi tradizionale di costanti discontinuità.

\section{I1 Mondo in quanto Universo.}

In effetti il passato del mondo è l'immagine che la storia dà di sé, ma in ciò non rivela una sorta d'intenzionalità husserliana: non si tratta di alcuna trascendenza della società o della storia. Per Sartre ${ }^{10}$ la coscienza non poteva trascendersi "né verso il mondo, né verso l'avvenire, né verso il passato" e con ciò muoveva proprio la sua critica a Husserl.

Eppure la coscienza permane esperienza trascendentale: il mondo è in-coscienza della sua storia futura che non ha ancora avuto essere, e successiva consapevolezza della possibilità del suo esserci solo come elemento discreto. Tale elemento discreto, tale possibilità dell'esserci interruzioni e discontinuità nel Tempo, cosa che la temporalizzazione evidenzia quale materiale forma di cesura tra un ente passato e un eventuale "recente" futuro, indica la trasformazione del mondo nel suo divenire nel Tempo come particolare momento del senso del mondo stesso presente all'avvenire della sua storia.

D'altronde il mondo emana luce del passato nella sua trasformazione, essendo costantemente inerente alla sua memoria, e riproduce questa sua memoria specificamente nella

${ }^{9}$ Citato da J.P. SARTRE, cit., p. 160.

${ }^{10} \mathrm{Si}$ vedano le articolate analisi ancora di J.-P- SARTRE, cit., pp. 27-28. 
trasformazione affermandola nel momento della sua tradizione. Così lo stesso futuro del mondo si traduce in realtà nella condizione del suo possibilizzarsi nel Tempo perché, onticamente, avvenga il suo peculiare presente: cioè condizione di passato rispetto al futuro e condizione di futuro rispetto al passato ${ }^{11}$.

Per cui l'onticità del mondo, cioè il suo proprio e specifico essere ermenenticità - la possibilità che si crei una molteplicità di interpretazioni probabili - fonda ogni segmento della ricostruzione della sua forma nel tempo del mondo, nella temporalità della storia perché la temporalizzazione riveli la trasformazione ontologica del mondo nella ricostruzione della storia del mondo quale larghezza del tempo, appunto sua temporalizzazione.

Peraltro le diverse caratteristiche di un sistema, il rapporto tra Tempo e Mondo, il movimento del mondo nel suo spazio e tempo, che vengono a essere unite e ricomprese rispetto al Tempo di evoluzione dello stesso concetto mondo, qualora fossero successivamente mediate rispetto a tutti i possibili stati del sistema Tempo-Mondo in un determinato momento proprio del Tempo, offrirebbero sì la validità della K-tempo nel mondo, ma lascerebbero anche intravedere come il Tempo medio di ritorno del mondo alla sua tradizione, dunque verso un suo stato stazionario, sia talmente grande da oltrepassare il Tempo di esistenza del

\section{Mondo $\Leftrightarrow$ Universo}

nel quale il concetto mondo evolve, come se appunto si annullasse il Tempo stesso nelle sue specifiche Temporalità, risolvendosi unicamente in Temporalizzazione: cioè in presente del mondo.

Proprio nel noto teorema di Jules-Henry Poincaré secondo cui nella stessa evoluzione di un sistema, all'incirca per ogni suo stato iniziale, il sistema ritornerebbe infinite volte a uno stato arbitrariamente vicino a quello iniziale, si evince come la trasformazione delle ermeneuticità del mondo potrebbe coincidere con i suoi successivi spazi temporali, prima ancora dei mutamenti del suo significato più proprio.

La questione, in effetti, investì già nell'antichità quanto venne a essere individuato come problema del passaggio (limen) dal limite (limes) a quello della continuità, che qui

\footnotetext{
${ }^{11}$ Dice Aristotele in Fisica, cit., p. 321: "E dal momento che è possibile che esista e possa essere pensato il tempo senza l'istante, mentre l'istante è qualcosa di intermedio, nello stesso tempo principio e fine - principio del futuro e fine del passato -, di necessità allora il tempo esisterà sempre; infatti l'estremità dell'ultimo tempo considerato, sarà nell'istante: non è infatti possibile assumere nel tempo null'altro che l'istante", cioè appunto il solo presente nella cui temporalità tutte le altre temporalità confluiscono come dimensione del Tempo e condizioni determinate del Tempo nel mondo.
} 
s'inserisce quale semantica che attraversa e solca il Tempo del mondo nel suo Spazio, tra un limite di Temporalità e la continuità ermeneutica del senso del concetto nella Temporalizzazione $^{12}$ : ci si ricollega a una delle dicotomie fondamentali della nostra esperienza: quella appunto del continuo e del discreto.

Non si può certo trascurare il fatto che già Bernhard Riemann ${ }^{13}$ avesse sostenuto come attraverso la teoria delle grandezze continue, nello studio delle funzioni ottenute mediante l'integrazione di differenziali esatti, si venissero a effettuare astrazioni da qualsiasi idea e misura, studiando soltanto i rapporti di posizione e di inclusione. Si potrà in virtù di ciò affermare che nella tradizione, intesa quale sviluppo del Tempo nella sua efficacia di Temporalizzazione del mondo, si rivela la qualità necessaria e il presupposto sufficiente mediante i quali stabilire l'unità di ogni trasformazione del mondo nel divenire quest'ultimo universale: in ciò ogni mutamento acquisisce effettività ontica, perché modello di una forma del senso che rivela il fenomeno storico del significato del mondo.

I successivi e progressivi emendamenti temporali, allora, costituiscono del mondo una forma continua da un punto di vista ontico ${ }^{14}$, possibile nella $K$-Tempo, da cui si otterranno quelle trasformazioni del significato del concetto che lasceranno fluttuare le possibili interpretazioni del mondo, sino a ricondurre il Tempo stesso all'unica e possibile tradizione del mondo. È come se il Tempo attraverso ogni trasformazione, che in esso si configura, testimoniasse costantemente la memoria del mondo. Si potrà perciò asserire che ogni trasformazione sia limite da un lato e serie, dall'altro lato, kata-strofici, interpretabili cioè come legami storici recisi e rotture epistemiche del mondo. Da tali legami e da tali cesure appare la singolarità fenomenica del mondo, o meglio si profilano le sue stesse fenomeniche che fondano l'Universale del mondo quale Fenomeno, prospettato attraverso una Forma che ricompare continua, segmento per segmento, costante e perenne nella tradizione.

\section{La scelta irrazionalmente esistenziale: Dio.}

La filosofia del tempo si traduce, quindi, in un'osservazione dell'ordine del mondo nel Tempo di questo mondo governato da Dio che è eterno, giacché né l'inizio né la fine, di questo

\footnotetext{
12 Cfr. M. HEIDEGGER, cit., p. 909 e ss. in riferimento al problema ontologico della trasformazione del Tempo nel mondo.

13 Cfr. B. RIEMANN, Lehrsätze aus der analysis situs für die Theorie der Integrale von zweigliedrigen vollständigen Differentialien, in "Journal für reine und angewandte Mathematik", XLIV, p. 105.

14 Cfr. L. WITTGENSTEIN, Tractatus logico-philosophicus, 2.022: "È manifesto che un mondo, per quanto differente sia pensato dal mondo reale, deve avere in comune con il mondo reale qualcosa: una forma".
} 
mondo, ci è dato conoscere. E per eterno non si può che intendere proprio ciò di cui si perde concetto, poiché non si perviene a cogliere quando il Tempo abbia avuto inizio nel segnare il movimento dell'universo, né potendo stabilire la durata di questo stesso Tempo, cioè efficacemente poter rispondere al quesito: quanto tempo resterebbe ancora al... Tempo ${ }^{15}$

Nell'ambito quindi di tale irrisolvibile indecidibilità si perviene alla concretezza dello spirito ermeneutico aristotelico che segna il Tempo pervaso dal prima e dal poi, successiva acquisizione in Agostino, il quale constata tuttavia la percezione di Dio non poter essere più accantonabile e separabile dalla vita dell'uomo e dalla sua "esistenza".

Proprio intuendo obiettivamente la vita come cammino, come destino (in spagnolo assume proprio il significato di meta, di arrivo) che, suo malgrado, deve raggiungere un traguardo paradossale nel suo opposto, la vita stessa colta e interpretata in esistenza, rivela l'antinomia del Tempo essere esplicazione del dilemma teologico: credo?

In sostanza la fede acquisisce certezza nel momento in cui si è altrettanto sicuri di non poter mai decidere - come quando si decide nella quotidianità, scelte razionalmente esistenziali -, proprio perché Dio è una scelta irraz̧ionalmente esistenziale, allo stesso modo di come la vita è paradossalmente legata in maniera inscindibile al suo opposto, la morte. Eppure è esattamente in questo opposto della vita che si afferra il suo riconoscimento quale realtà incontrovertibile che esiste solo, e solo se, non-morta.

L'accadimento più irrazionale nella vita, cioè il "curioso" verificarsi del suo contrario, necessita esistenzialmente il mistero di Dio, legittima ontologicamente proprio la vita dell'uomo, permette mondanamente uno sguardo sul Tempo, quasi che la morte fosse la "carta d'identità" della vita. Ecco perché il mistero della salvezza in Cristo libera definitivamente l'amore verso di Lui, amore costretto a lottare contro la prigione dell'esistenza per testimoniare la sua vita, e che è sciolto dalle catene solo quando il paradosso torna alla sua ricomposizione finale ${ }^{16}$.

15 Cfr. K. GÖDEL, Über formal unentscheidbare Sätze der "Principia Mathematica" und verwandter Systeme, in "Monatshefte für Mathematik und Physik", XXXVIII, (1931); da non ovviare alle analisi di S. J. GOULD, Ontogeny and Phylogeny, The Belknap Press of Harvard University Press, Cambridge (Mass.) 1977, in particolar modo pp. 34-41; si veda anche J. GUITTON, Che cosa credo, Bompiani, Milano 1993, quando l'autore a p. 84 afferma: 'L'errore moderno, a mio avviso, consiste nel credere che 'il problema di Dio' riguardi solo la fede, il sentimento o la scommessa. In realtà, esso si pone alla ragione nel momento in cui è totale e pura, quando raccoglie tutte le sue forze, quando è pienamente se stessa - cosa, questa, così rara e sicuramente furtiva e momentanea...".

16 In effetti la paradossalità del morire consiste proprio nella sopravvivenza di quanto esterno al morire per sé. Dice Martin Spaeth: 'Die Welt 'überlebt' den Menschen, so dass ein Versagungsgefühl aufkommt, also das äußerst missliche Empfinden, ihm werde durch den Tod etwas vorenthalten, auf das er womöglich ein Anrechte habe”. In questo modo s’indirizza M. SPAETH, Gewonnene Zeit-verlorenes Heil?, LIT, Berlin 2007, p. 142. 
A tal proposito nella Scienza della Croce Edith Stein, filosofa martire, parla di Dio come di un mistero che avvolge il nostro proprio essere, perché è nell'anima di ognuno che Dio si riserva di divenire, nella presenza di Cristo e nellinconsumabile anelito che lega ognuno di noi all'Immortalità. Questo mistero è svolto dalla Stein come resurrezione della carne che si eleva dal Verbo per assurgere ad opera santificata della vita dell'uomo. Il mistero dell'essere avvolge l'anima di ognuno come un involucro donato da Dio che noi possiamo aprire verso l'Altro, per cogliere nella congiunzione terrena il verso poetico tale da consentire il divenire in un $A l$ di là eterno e privo di corpo.

Così ogni tratto della forma del mondo è movimento e misura del Tempo che ravvolge il Creato, perché l'uomo ne sia partecipe come dimensione di colui-creato. L'anima stessa trova perciò la consapevolezza del ritorno celeste soltanto conoscendo e amando Dio: la Stein ci dice chiaramente che l'anima acquisisce possesso e intensità della sua dimensione creata quando si unisce ed è in corrispondenza con Dio.

Questa condizione, che non è sempre comune tra gli uomini, è però vera sempre per l'uomo, se quest'ultimo ponga ineludibile nella sua meditazione la tendenza a precipitarsi in un centro - come sostiene ancora Edith Stein - effettivamente in grado di condurre l'uomo a contatto con il suo Creatore. Cristo stesso nell'ora del Getsemani vive la profondità della sua meditazione nel farsi raggiungere dalla volontà paterna, cui riconosce tutto, anche la sofferenza che di lì a poco tormenterà il suo corpo e la sua anima, anticipando la Croce $^{17}$.

L'amore, in effetti, riuscirà a essere e a divenire, se consapevole della sua libertà nella felicità che è ritrovata soltanto e unicamente nel volersi immolare e donare. Così l'umanità della morte riaffiora in un anelito al divino, in una primigenia fonte che riconduce l'essere a poter ancora volere, nell'istinto dell'anima verso il Creatore, e desiderare la dimensione di una finalità espressa per la riunione di ogni elemento terreno che assurge a consistenza celeste.

A tal proposito dice Jean Guitton: "Ama Dio è un richiamo verticale; ama il tuo prossimo è un richiamo orizzontale. Queste due vie sono identiche, dato che Dio è invisibile. E non possiamo sapere se lo amiamo: è l'amore che portiamo verso l'altro che ce ne fornisce la prova. Questo è il pensiero che esprimeva san Giovanni, per illustrare quello del suo maestro. Ma solo coloro che credono, che pregano e che adorano possono compiere l'atto verticale. Un ateo ama semplicemente l'uomo"'18.

17 "La sofferenza di Gesù nel Getsmani fu di tale entità ed eccezionalità da provocare questo raro fenomeno: sudore di sangue. Sangue di Gesù che affiora sul suo volto e cade in terra; sangue che è un'altra - o l'identica manifestazione del rinnovato 'sì' di Gesù al piano di redenzione del Padre e che si sparge già a remissione dei nostri peccati, anticipando la Croce”, così J. ECHEVARRIA, Getsemani, Ed. Ares, Milano 2008, p. 247.

${ }^{18}$ Così J. GUITTON, cit., p. 46. 


\section{Conclusioni: la rivelazione di Dio in Cristo.}

Tuttavia amare il prossimo significa amarlo nella visione di un amore verticale che proviene e che si dirige a Dio, amore divino che nella meditazione è dato scoprire totalmente e infinitamente: "Dio può concedere all'anima un'oscura e amorosa conoscenza di Se stesso, anche senza quell'allenamento preparatorio che è la meditazione. Egli può portarla di peso, improvvisamente, nello stato di contemplazione e d'amore, vale a dire infonderle la contemplazione" ${ }^{\prime 19}$.

Tale questione fu ripresa da Fernando Inciarte che non mancò di sottolineare come nell'aspetto metafisico dell'anima si riveli palesemente l'orizzonte del divenire la stessa anima, concetto-guida nell'analisi esistenziale dell'uomo e della sua storia ${ }^{20}$.

Nella morte, peraltro, è la fede che realizza la vita come un evento della grazia, perché se la fede muove il ritorno a Dio, quotidianamente, neppure si può dubitare come, a sua volta, sia la grazia di Dio ad imprimere alla vita dell'uomo la necessità della fede di fronte al mistero della sua vita. E proprio Gesù, cioè la rivelazione di Dio nel Tempo del mondo, soffre il mistero della necessità della salvezza del mondo attraverso quanto si oppone al potere politico, al dominio "semplice" del tiranno che saccheggia e umilia, infine attraverso quanto si oppone alla morte, cioè l'amore, quell'amore già narrato forte come la morte ${ }^{21}$, ma che tuttavia necessita della morte per essere e per rivelarsi e per vivere, perché è in quell’istante in cui la vita soffre tale necessità del mistero del suo proprio essere che la stessa vita finalmente risorge quale fulcro di pienezza della salvezza e diviene, la vita, evento nel mondo, contemporaneità assoluta nell'universo senza inizio e senza fine.

Da ciò si evince la possibilità di cogliere Dio in un solo impercettibile istante, istante in cui Dio ha sofferto il mistero della sua necessità di oltrepassare l'apparente inaccessibile angoscia, al fine di vincerla a dispetto del pericolo della debolezza di essersi potuto rivelare politicamente, come capo per il dominio della storia e sul mondo.

\footnotetext{
${ }^{19}$ Cfr. E. STEIN, Scientia Crucis, Ed. OCD, Roma 1998, p. 205. Cfr. di F. INCIARTE, Unsterblicbkeit der Seele und Auferstehung des Leibes, in Leben zur Gänze. Das Leib-Seele-Problem, vol. 7, Religion, Wissenschaft, Kultur, Schriftenreihe der Wiener katholischen Akademie, ed. G. Pöltner, H. Vetter, Präsidium der Wiener Katholischen Akademie, Wien/ München, Herold, 1986, p. 82-95.

${ }^{20}$ Su questi profili si veda di F. INCIARTE, Die Seele aus Begriffsanalytischer Sicht, in Entstehung des Lebens, Studium generale WS 1979/80, H. Seebass (Hrsg.) Schriftenreihe der Westfälischen Wilhelms-Universität Münster, Heft 2, Aschendorf, Münster, 1979, p. 47-70.

${ }^{21} \mathrm{Ct}, 8,6-7$.
} 
È invece nella salvezza del dolore che Cristo manifesta e dis-vela tutta la sua bellezza di essere Figlio di Dio, bellezza solo apparentemente e momentaneamente sconfitta dalla morte, bellezza però che risorge in quanto amore di quella Vita che accade ancora nel Tempo perché memoria del mondo ${ }^{22}$.

La realtà poetica che il mondo ci descrive attraverso la continuità del Tempo, s'incammina verso il destino, per ognuno inevitabile, di una storia ai cui bordi, probabilmente veri e propri cigli di tanti possibili precipizi in agguato, l'uomo osserva, malinconicamente, quanto è ormai stato irrimediabilmente perduto o quanto diviene inaccessibile.

D'altronde quando Heidegger ritenne di dover cogliere nell'essenza dell'esistenżalità, l'avvenire come peculiarità di tale essenza, non fece altro che scoprire quel velo che ravvolge il mistero del Tempo nel mondo e della vita nel mondo quale accadimento che trascende la temporalità (die Zeitlichkeit) e la temporalizzazione (die Veržeitlichung): appunto perché il Tempo originario è finito, e quello derivato acquisisce la specificità dell'in-finito, attraversando interamente la vita dell'uomo, all'interno del Tempo nel mondo. L'uomo così diviene il suo stesso avvenire ultramondano, perché ha solcato il mondo con la sua vita ed è peculiarmente in questo che l'uomo ha mondanizzato il mondo, l'ha cioè reso-vissuto nell'elemento discreto del Tempo sino a consegnare alla grazia tutto quanto avrebbe potuto aspettarsi dal suo stesso avvenire.

E la vita acquisisce così il suo... senso.

Allo stesso modo, su tale prospettiva, essendo l'universo "vivente", lo è nella misura in cui possiede un'anima, cioè il suo tempo, la sua durata, la misura del suo Spazio, tanto infinita quanto intuibile concreta unicamente in Dio, Tempo e anima, misura e sospensione dell'intero universo ${ }^{23}$.

In questo "universo vivente" si coglie la congiunzione tra cosmologia e ontologia, quasi a dover necessariamente ammettere entrambe frutto della molteplicità dell'essere e della conseguente variabilità del divenire, forma e fenomeno del moto del Tempo nel mondo e di quest'ultimo nello Spazio illimitato e indeterminabile, coniugazione tra l'eterno di Dio e l'in-finito

\footnotetext{
22 Dice Javier Echevarría: "Non riusciremo mai a farci carico pienamente del male che noi uomini abbiamo commesso nel corso della storia. Gesù, che percepisce in tutta chiarezza quella mole di immondizia scagliata contro Dio, si sprofonda esterrefatto perché la sua perfezione di intelligenza e di amore lo porta a penetrare tutta la degradazione alla quale noi creature abbiamo condisceso. Ma a tanta malvagità, che lo colpisce nell'anima e nel corpo con una sofferenza indescrivibile, risponde con quella pienezza di amore, così immensa da cancellare quella progressione di miserie: Uomo, $i$ tuoi peccati ti sono rimessi $(L c, 5,20)$ ", così J. ECHEVARRIA, cit., pp. 219220.

${ }^{23}$ Cfr. A. JORI, Der Kosmos als Lebewesen. Einige Probleme und Lösungen des 'astronomischen Vitalismus' in Aristoteles, De Coelo, in AA. VV., Antike Naturwissenschaft und ibre Rezeption, a cura di A. Althoff, B. Herzhoff e G. Wöhrle, vol. XII, Trier 2002, pp. 69-86.
} 
dell'universo, perenne verso il dopo, come evinto sopra, dunque unicità di quella e solo di quell'immagine: il volto di Dio, che è forma in Gesù, e diviene fenomeno esattamente quando Gesù compie la rivelazione di Dio, rivelandosi egli stesso Dio.

Sicché nel Primo Moto Immobile (Unbewegte Grundbewegung), moto primo senza inizio del Tempo nel mondo ma che ha dato inizio al Tempo continuo del mondo, immobile perché in sé origine di ogni movimento, in quanto per-sé non soggetto alla misura della durata, dunque indefinibilità di movimento eterno, si concentra il movimento iniziale che ha lasciato svolgersi ogni res, restando appunto immobile, proprio perché non soggetto a mutamento o a cambiamenti, modello senza limite eppur perfetto, indeterminabile nel Tempo perché creatore di ogni tempo, continuo nello Spazio illimitato e indeterminato, sino alla possibilità di enucleare un continumm universale: Dio, misura del tempo nella via e nella vita dell'uomo per essere quest'ultimo in Dio e divenire, nel senso di accadere, così, uomo. 
Riferimenti bibliografico

ARISTOTELE. Fisica. Milano: Mimesis Edizioni, 2007.

BLUMENBERG, H. La leggibilità del mondo. Bologna: Il Mulino, 1984.

ECHEVARRIA, J. Getsemani. Milano: Ed. Ares, Milano 2008

GÖDEL, K. Über formal unentscheidbare Sätze der "Principia Mathematica" und verwandter Systeme. Monatshefte für Mathematik und Physik, XXXVIII, 1931.

GOULD, S. J. Ontogeny and Phylogeny. Cambridge: The Belknap Press of Harvard University Press, 1977.

GUITTON, J. Che cosa credo. Milano: Bompiani, 1993.

HEIDEGGER. Essere e tempo. Milano: Mondadori, 2006.

INCIARTE, F. Die Seele aus Begriffsanalytischer Sicht. In: Entstehung des Lebens. Aschendorf, Münster: Studium generale, 1979.

INCIARTE, F. Unsterblichkeit der Seele und Auferstehung des Leibes. In: G. Pöltner, H. Vetter (ed.). Leben zur Gänze. Das Leib-Seele-Problem. Präsidium der Wiener Katholischen Akademie, Wien/ München: Herold, vol. 7, 1986.

JORI, A. Der Kosmos als Lebewesen. Einige Probleme und Lösungen des 'astronomischen Vitalismus' in Aristoteles, De Coelo. In: AA. VV. Antike Naturwissenschaft und ihre Rezeption. Trier, vol. XII, 2002.

LONGO, G. Trattato di semantica storica e di logica giuridica costituzionale. Roma: Aracne, 2009.

RIEMANN, B. Lehrsätze aus der analysis situs für die Theorie der Integrale von zweigliedrigen vollständigen Differentialien. Journal für reine und angewandte Mathematik, vol. 1857, n. 54, p. 105-110, 2009.

SARTRE, J.- P. L'essere e il nulla. Saggio di ontologia fenomenologica. Milano: Il Saggiatore, 1984.

SPAETH, M. Gewonnene Zeit-verlorenes Heil? Berlin: LIT, 2007.

STEIN, E. Scientia Crucis. Roma: Ed. OCD, 1998.

WITTGENSTEIN, L. Tractatus logico-philosophicus. Torino: Giulio Einaudi, 2009. 